\title{
Indoor radio channel modeling and mitigation of fading effects using linear and circular polarized antennas in combination for smart home system at $868 \mathrm{MHz}$
}

\author{
S. Wunderlich, M. Welpot, and I. Gaspard \\ Hochschule Darmstadt, Department of Electrical and Computer Engineering, Darmstadt, Germany \\ Correspondence to: I. Gaspard (ingo.gaspard@h-da.de) \\ Received: 9 December 2013 - Revised: 29 May 2014 - Accepted: 22 June 2014 - Published: 10 November 2014
}

\begin{abstract}
The markets for smart home products and services are expected to grow over the next years, driven by the increasing demands of homeowners considering energy monitoring, management, environmental controls and security.

Many of these new systems will be installed in existing homes and offices and therefore using radio based systems for cost reduction. A drawback of radio based systems in indoor environments are fading effects which lead to a high variance of the received signal strength and thereby to a difficult predictability of the encountered path loss of the various communication links.

For that reason it is necessary to derive a statistical path loss model which can be used to plan a reliable and cost effective radio network. This paper presents the results of a measurement campaign, which was performed in six buildings to deduce realistic radio channel models for a high variety of indoor radio propagation scenarios in the short range devices (SRD) band at $868 \mathrm{MHz}$.

Furthermore, a potential concept to reduce the variance of the received signal strength using a circular polarized (CP) patch antenna in combination with a linear polarized antenna in an one-to-one communication link is presented.
\end{abstract}

\section{Introduction}

Indoor radio channels suffer usually from slow fading. That means that the characteristics of the channel vary only slowly in time compared with the duration of a data symbol. This can become a very dramatic effect in the communication between two (or more) radio devices. Especially if these devices are non-mobile devices, like in a smart home system.
The reason for that is, that if the receiver is located in a deep fade it will probably remain in this deep fade for a long (or unlimited) time period (Vireerackody, 1993). A deep fade can significantly decrease the signal-to-noise ratio (SNR) of a communication link, which results in a reduced data rate or in a complete drop out of the radio link.

Another effect which can also cause deep fades is fast fading. Fast fading occurs in indoor scenarios due to moving obstacles such as moving people (Horvat et al., 2013). In a fast fading channel, the transmitter may take advantage of the variations in the channel conditions using time diversity (which is often used by smart home systems). However, this paper does not cover the influences of moving people and objects on the radio channel, for further information on this topic, see e.g. Horvat et al. (2013).

The main reason for fading in indoor environments is multipath propagation. Transmitted radio waves are reflected and scattered on walls, ceilings, floors and other obstacles and can combine at the receiver in a destructive manner, due to different phase shifts and amplitudes of the arriving signal paths (Molisch, 2011). Another effect is depolarization. The scattered and reflected waves that contribute to multipath fading can also transfer energy from the transmitted polarization plane into the orthogonal polarization plane (called cross-polarization coupling) (Cox et al., 1986). Such coupling occurs as a result of oblique reflections from the walls as well as due to scattering from indoor clutter, such as furniture (Kyritsi and Cox, 2001). This effect can cause significant degradation in signal quality if the transmitting and receiving antennas are using the same polarization. This effect contributes also to fading (polarization fading).

Since the delay spread of indoor channels is in the range of nanoseconds (Hashemi and Tholl, 1992) and the bandwidth 


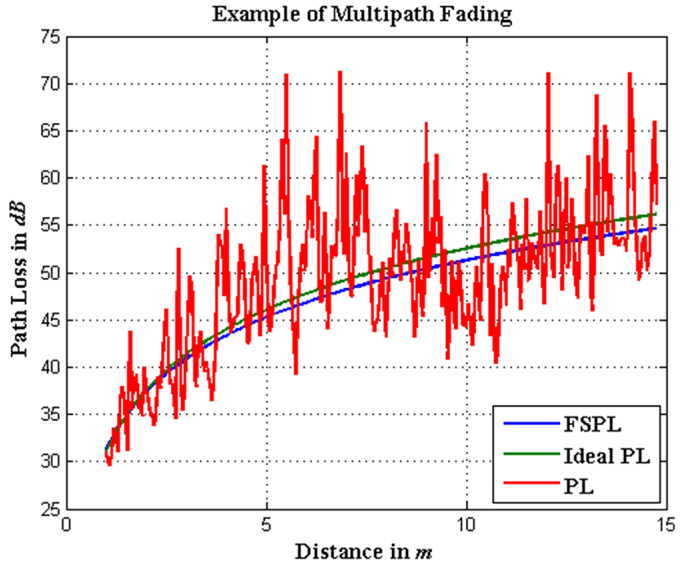

(a)

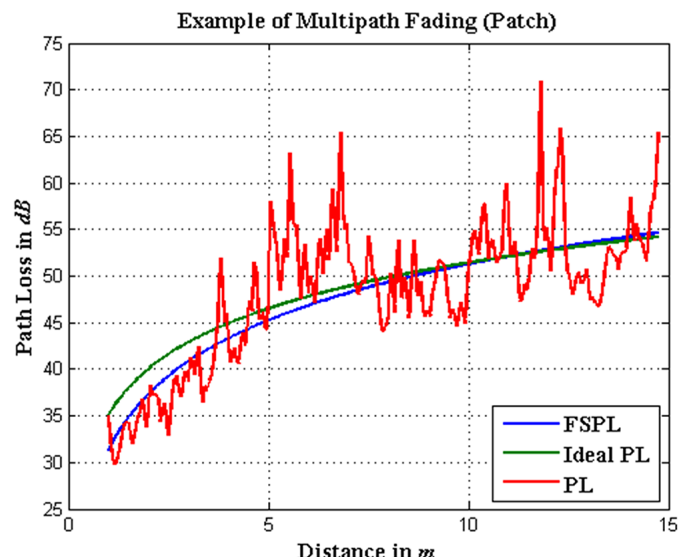

(b)

Figure 1. (a) A channel encounters strong fading using two vertical linear polarized antennas. The red graph (PL) represents the actual path loss the channel encounters (which was measured during the measurement campaign), the blue graph (FSPL) represents the theoretical free space path loss (for reference) and the green graph (Ideal PL) represents the actual path loss the channel encounters without fading effects. This graph was created by using formula 5 with a minimum mean square error (MMSE) approach to calculate the path loss exponent based on the original data set (red graph). (b) Mitigation of fading using a CP antenna in interaction with a vertically linear polarized antenna.

of the most indoor devices is relatively small (the SRD bandwidth at $868 \mathrm{MHz}$ is below $600 \mathrm{kHz}$ ), the channel can be assumed to show flat fading, so the coherence bandwidth is much larger than the signal bandwidth and the channel does not show a frequency-selective behavior.

These fading effects can lead to a significant reduced signal quality between sender and receiver and represent a significant problem for radio communication in indoor transmission scenarios.

One way to mitigate fading introduced by crosspolarization coupling is to use a circular polarized antenna in interaction with a linear polarized antenna. The benefit of using a circular polarized antenna at one end of the communication link is the irrelevance of the orientation angle of the polarized wave, so the $\mathrm{CP}$ antenna can receive radio waves at an arbitrary polarization plane and the effect of crosspolarization coupling can be annihilated. The only drawback of this method is the additional $3 \mathrm{~dB}$ polarization loss introduced by using a circular polarized antenna in combination with a linear polarized antenna. One example of the reduction of fading using a circular polarized antenna in comparison to a vertical linear polarized antenna can be seen in Fig. 1. So this method is based on the idea that all values are decreased by $3 \mathrm{~dB}$ but the mean value of the received power level is much higher than without this method. This is due to the fact that the influence of the fading spikes is drastically reduced.

The variance of the received power in case of two linear polarized antennas is obviously much larger than in case of using one linear and one circular polarized antenna for the same radio link. Another positive effect of using a CP antenna is that the orientation of the linear polarized antenna does not influence the communication link anymore. This is particularly advantageous since the orientation of the anten- nas can seldom be guaranteed for the most indoor application scenarios. This approach is especially practical if a masterslave relationship between a central radio module and one or more radio subunits is given (like in centralized smart home systems). The central radio module can use the $\mathrm{CP}$ antenna while the minor radio devices still use low-cost and spacesaving linear polarized antennas.

To verify this theoretical concept a measurement campaign in six buildings was performed (see Sect. 4).

So the two main objectives of this paper are:

1. Presentation of the results of a potential fading mitigation technique using a circular polarized patch antenna in combination with a linear polarized antenna. This antenna is subject to further improvements regarding size and cost reduction. The antenna itself is a well-known design (truncated-corner square patch), however the advantage of using a circular polarized antenna at one end of the link and a linear polarized antenna at the other end of the link in comparison to the conventional usage of linear polarized antennas at both ends should be proven on the basis of a comprehensive measurement campaign.

2. Development of a path loss prediction model which covers a versatile number of scenarios and can be used for a simple estimation of the expected path loss in an actual smart home installation. There does not exist many of these prediction models for this specific frequency domain which are based on an extensive measurement campaign. 


\section{Measurement setup and procedure}

\subsection{Measurement setup}

The measurement setup is depicted in Fig. 2. The transmitting part of the setup is shown on the left side of the figure. The transmitting antenna is a cross polarized log-periodic antenna which is used to generate a horizontal and vertical polarized electromagnetic wave (denoted with XSLP9142, Schwarzbeck Mess-Elektronik, 2013). The circular polarized patch antenna can be used as an alternative transmitting antenna.

The two input ports of the cross polarized antenna are fed by two carrier wave (CW) generators at $873.8 \mathrm{MHz}$ and $874 \mathrm{MHz}$ (The prototype antenna was designed for a resonance frequency at $f=873.9 \mathrm{MHz}$ and not for the actual SRD frequency at $f=868.3 \mathrm{MHz}$.) at an output power of $P_{\mathrm{CW}_{\mathrm{dBm}}}=14 \mathrm{dBm}$.

These slightly different frequencies were chosen to distinguish between the two polarizations states in the spectrum which were received by a circular polarized (CP) patch antenna (or a dipole antenna as reference) and measured by a spectrum analyser (SA) which stored it on a PC for further data processing.

The height of the phase center of the transmitting and receiving antenna was $h=1.16 \mathrm{~m}$. The transmitting antenna was located at a fixed position while the receiving antenna was moved along the measuring track during a single measurement run. A truncated-corner patch antenna design was used for the circular polarized antenna with a beamwidth of $\theta_{-3 \mathrm{~dB}} \approx 60^{\circ}$, an antenna gain of $g=-1 \mathrm{dBi}$ and a return loss of $\mathrm{RL}=30.8 \mathrm{~dB}$. The spatial resolution of a single measurement path was $d=0.05 \mathrm{~m}$ (distance between two measurement points) which results in a spatial sampling rate (SSR) of

$$
\begin{aligned}
& \lambda=\frac{c_{0}}{f}=\frac{c_{0}}{874 \mathrm{MHz}}=0.343 \mathrm{~m} \\
& \mathrm{SSR}=\frac{\lambda}{d}=\frac{0.343 \mathrm{~m}}{0.05 \mathrm{~m}}=6.86
\end{aligned}
$$

samples per wavelength which results in a sufficient detectability of fading spikes (where $\lambda$ is the wavelength, $c_{0}$ is the speed of light and $f$ is the operating frequency).

The path loss PL can be fairly simple measured by using the relationship:

$\mathrm{PL}=P_{\mathrm{Tx}_{\mathrm{dBm}}}-P_{\mathrm{Rx}_{\mathrm{dBm}}}$

where $P_{\mathrm{Tx}_{\mathrm{dBm}}}$ is the transmitted and $P_{\mathrm{Rx}_{\mathrm{dBm}}}$ is the received power. Taking into account antenna gains and cable losses it follows:

$$
\begin{aligned}
& \mathrm{PL}=\left(P_{\mathrm{CW}_{\mathrm{dBm}}}-a_{\mathrm{Tx}}+g_{\mathrm{Tx}}\right)-\left(P_{\mathrm{SA}_{\mathrm{dBm}}}+a_{\mathrm{RX}}-g_{\mathrm{Rx}}\right) \\
& \mathrm{PL}=P_{\mathrm{CW}_{\mathrm{dBm}}}-a_{\mathrm{Tx}}-a_{\mathrm{RX}}+g_{\mathrm{Tx}}+g_{\mathrm{Rx}}-P_{\mathrm{SA}_{\mathrm{dBm}}}
\end{aligned}
$$

where $P_{\mathrm{SA}}$ is the power at the spectrum analyzer and $a$ is the cable loss and $g$ is the respective antenna gain at $\mathrm{Rx}$ and Tx.

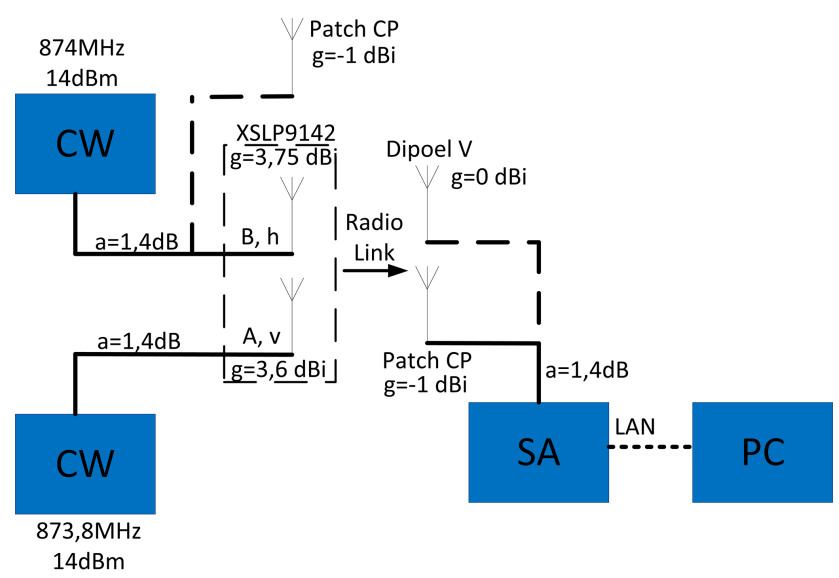

Figure 2. Measurement and test setup for the indoor radio channel modeling and the circular polarized patch antenna. $(\mathrm{SA}=$ spectrum analyser, $\mathrm{CW}=$ carrier wave generator).

\subsection{Measurement procedure}

The measurements were performed in six buildings on the campus of the Hochschule Darmstadt. All of these buildings differ in parameters like material, geometry, usage etc. So a huge variety of different fading scenarios was achieved (see Table 1).

Although all measurements were mostly taken in officelike environments there are some similarities between these environments and residential environments. For example the Building D1617 shows similar room geometry, building materials and furniture like many residential areas.

A section of a floor plan of one of the buildings can be seen in Fig. 3. The plan shows the entrance and the hallway of building B11. Basically two types of measurements were performed, line-of-sight (LOS) measurements within rooms and floors (denoted with yellow arrows) and non-lineof-sight (NLOS) measurements with walls, doors and furniture between transmitter and receiver (denoted with red arrows). The starting point and the direction of the single measurement runs were chosen randomly to guarantee an equal distribution of all possible propagation scenarios within the building.

\section{Path loss prediction model}

The log-distance path loss model was used for the path loss prediction model (Seidel and Rappaport, 1992). It is based on the assumption that the (mean) path loss (PL) is a function of distance $\mathrm{d}$ to the $\gamma$-th power

$\mathrm{PL} \sim d^{\gamma}$

where $\gamma$ is the path loss exponent which indicates how fast path loss increases with distance. A logarithmic distance is used in the actual model, so the exponent $\gamma$ becomes a factor 
Table 1. List of buildings which were used during the measurement campaign.

\begin{tabular}{|c|c|c|c|}
\hline $\begin{array}{l}\text { Building } \\
\text { (name) }\end{array}$ & Type & Building materials & Designated usage \\
\hline B11 & $\begin{array}{l}\text { One-story lightweight } \\
\text { construction building }\end{array}$ & Wood & Lectures and seminars \\
\hline B14 & $\begin{array}{l}\text { Two-storied shipping } \\
\text { container architecture }\end{array}$ & Metal and wood & Lectures and seminars \\
\hline $\mathrm{C} 10$ & $\begin{array}{l}\text { High-rise building (15 } \\
\text { floors) }\end{array}$ & Reinforced concrete & $\begin{array}{l}\text { Offices, lectures, semi- } \\
\text { nars and labs }\end{array}$ \\
\hline D11 & Industrial building & Bricks and metal & $\begin{array}{l}\text { Workshops, lectures } \\
\text { and labs }\end{array}$ \\
\hline D1617 & Office building (5 floors) & Bricks and drywalls & $\begin{array}{l}\text { Offices, seminars and } \\
\text { labs }\end{array}$ \\
\hline
\end{tabular}

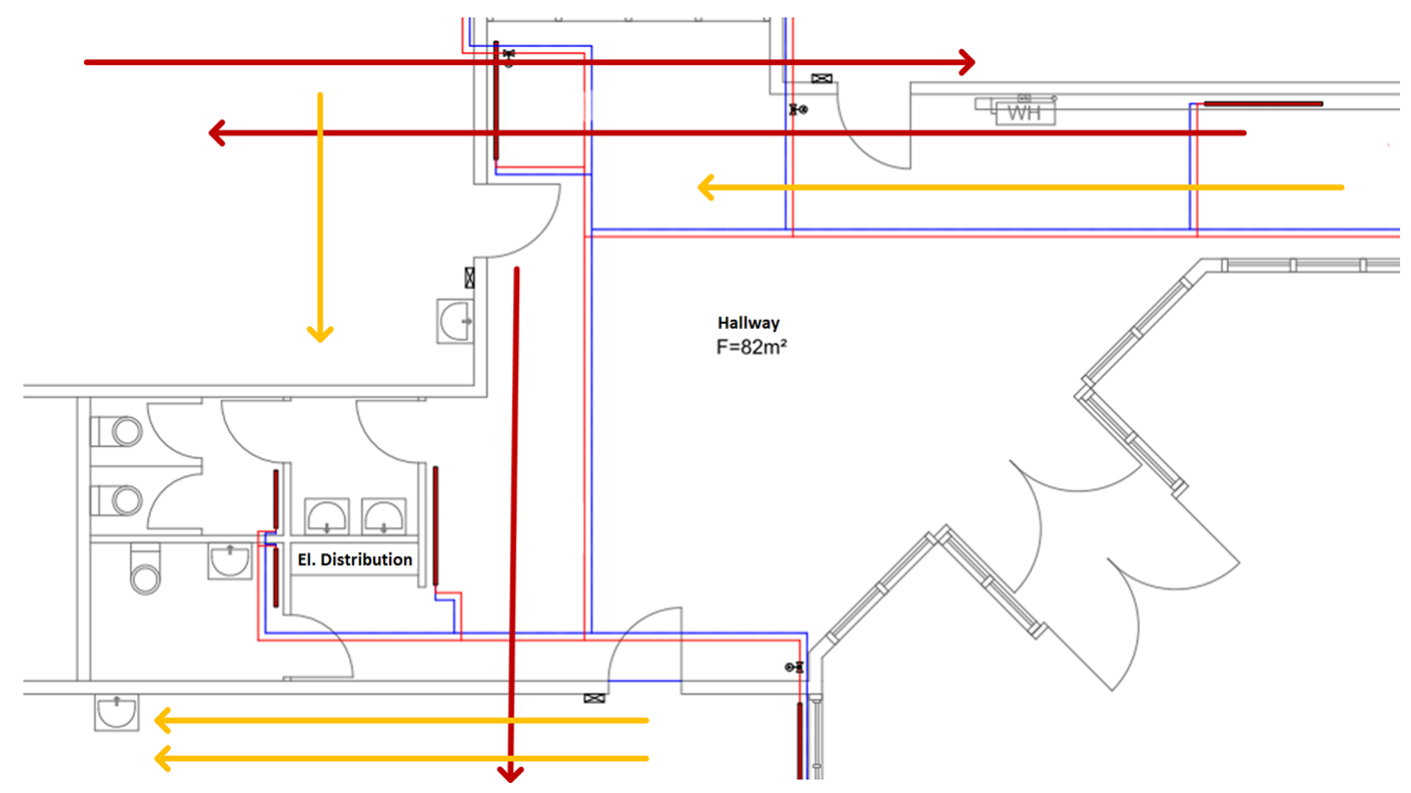

Figure 3. Section of a floor plan of one of the buildings (B11) used for the measurement campaign.

and the formula reads:

$\mathrm{PL}=\mathrm{PL}_{0}+10 \cdot \gamma \cdot \log _{10} \frac{d}{d_{0}}+X_{g}$

where $\mathrm{PL}_{0}$ is the path loss at a certain distance $d_{0}$ and $X_{g}$ is a normal distributed random variable which represents fading with zero mean and a standard deviation $\sigma$ in $\mathrm{dB}$. $\mathrm{PL}_{0}$ and $d_{0}$ represent the path loss from the transmitter to the distance $d_{0}$ where the measurement starts. $d_{0}$ can be freely chosen and was determined to be one meter for all LOS measurements $\left(d_{0}=1 \mathrm{~m}\right)$. The value of $\gamma$ is dependent on the propagation scenario of the measurement, it is two $(\gamma=2)$ for free space path loss (FSPL), greater than two $(\gamma>2)$ when the path loss increases faster than in free space and smaller than two $(\gamma<2)$ when it increases slower than in free space.

For a homogenous environment $\gamma$ can be assumed to remain constant for different distances between transmitter and receiver. However, if the propagation properties are changing (e.g. a wall separates transmitter and receiver) the path loss exponent $\gamma$ is also changing. For this reason it is appropriate to divide the measuring track into several separate segments. $\gamma$ can then be calculated separately for each of the segments. To compensate the additional path loss introduced by obstacles which influence the path loss exponent it is necessary to introduce new attenuation factors in Eq. (5) (Seidel and Rappaport, 1992):

$\mathrm{PL}=\mathrm{PL}_{0}+10 \cdot \gamma \cdot \log _{10} \frac{d}{d_{0}}+X_{g}+m \cdot \mathrm{FAF}_{\mathrm{dB}}+n \cdot \mathrm{WAF}_{\mathrm{dB}}$

where $\mathrm{FAF}_{\mathrm{dB}}$ represents a "Floor Attenuation Factor" and $\mathrm{WAF}_{\mathrm{dB}}$ means "Wall Attenuation Factor". $m$ and $n$ are representing the number of floors respectively walls which are penetrated by the radio wave (Seidel and Rappaport, 1992). This model is more flexible and more accurate than the 
Table 2. Path loss exponent $\gamma$ and standard deviation $\sigma$ in $\mathrm{dB}$ of fading for every building.

\begin{tabular}{|c|c|c|c|c|c|c|c|c|c|}
\hline Results & $\gamma$ & $\gamma$ & $\gamma$ & $\sigma$ & $\sigma$ & $\sigma$ & $\sigma$ & $\sigma$ & $\sigma$ \\
\hline Tx antenna & $\mathrm{V}$ & $\mathrm{H}$ & $\mathrm{CP}$ & V & $\mathrm{H}$ & $\mathrm{CP}$ & V & $\mathrm{H}$ & $\mathrm{CP}$ \\
\hline Propagation & & & & LOS & LOS & LOS & NLOS & NLOS & NLOS \\
\hline B11 & 1.59 & 1.95 & 1.8 & 3.18 & 4.54 & 2.51 & 5.28 & 4.91 & 11.60 \\
\hline B14 & 1.94 & 1.70 & 1.71 & 4.59 & 4.11 & 4.58 & 5.45 & 5.19 & 7.25 \\
\hline $\mathrm{C} 10$ & 1.69 & 1.56 & 1.65 & 4.84 & 5.00 & 4.86 & 7.93 & 6.94 & 5.63 \\
\hline D11 & 1.99 & 2.07 & 2.03 & 3.86 & 4.28 & 3.3 & 5.50 & 5.50 & 4.15 \\
\hline D1617 & 1.95 & 2.3 & 2.17 & 4.3 & 5.02 & 3.67 & 5.33 & 5.12 & 6.01 \\
\hline Average Value & 1.83 & 2.00 & 1.90 & 4.21 & 4.68 & 3.83 & 5.62 & 5.39 & 5.98 \\
\hline
\end{tabular}

Table 3. Standard deviation $\sigma$ in $\mathrm{dB}$ of fading in dependency of the used receiver antenna. $\mathrm{Rx}$ antenna: $\mathrm{V}=$ vertical polarized dipole, $\mathrm{CP}=$ circular polarized patch antenna.

\begin{tabular}{lllllllllll}
\hline Rx antenna & $\mathrm{V}$ & $\mathrm{V}$ & $\mathrm{CP}$ & $\mathrm{CP}$ & $\mathrm{CP}$ & $\mathrm{V}$ & $\mathrm{V}$ & $\mathrm{CP}$ & $\mathrm{CP}$ & $\mathrm{CP}$ \\
Tx antenna & $\mathrm{V}$ & $\mathrm{H}$ & $\mathrm{V}$ & $\mathrm{H}$ & $\mathrm{CP}$ & $\mathrm{V}$ & $\mathrm{H}$ & $\mathrm{V}$ & $\mathrm{H}$ & $\mathrm{CP}$ \\
Propagation & LOS & LOS & LOS & LOS & LOS & NLOS & NLOS & NLOS & NLOS & NLOS \\
\hline$\sigma / \mathrm{dB}$ & 4.90 & 5.40 & 3.52 & 3.96 & 3.83 & 6.00 & 5.67 & 5.52 & 5.12 & 5.98 \\
\hline
\end{tabular}

conventional log-distance path loss model (Hernandez et al., 2012), since the hard-to-predict influences of walls and floors on the path loss is shifted in separate summands. So the pathloss exponent becomes only dependent from the geometry of the room. All later presented results show only the path-loss exponent and not the FAF and WAF values since there was not enough data to calculate these values for single walls and floors. However, since the path-loss exponent is calculated separately for every segment between walls (or floors) there is no need to calculate these values ( $d_{0}$ was chosen according to the actual distance between sender and starting point for NLOS measurements).

\section{Results}

The results of over 13000 measuring points and 270000 single measurements were recorded and processed to obtain a general fading model of the investigated buildings and an adequate data set to study the effects of the optimized circular polarized antenna in combination with a linear polarized antenna on fading.

Table 2 shows the cumulated results of the measurement campaign separated for every building. The first three columns are representing the path loss exponents for a vertically $(\mathrm{V})$, horizontally $(\mathrm{H})$ and circular polarized $(\mathrm{CP})$ transmitted wave.

The last six columns representing the standard deviation of fading for a LOS and NLOS connection between transmitter and receiver. All results are mean values of the results of the two available receiving antennas: a vertical polarized dipole and the circular polarized patch antenna.

Some observations in Table 2 are remarkable:
1. The path loss exponent is smallest if a vertically polarized wave is transmitted. One possible explanation for that is that the walls in a building can be treated as dielectric materials. Horizontal polarized waves can penetrate the walls if the angle of incidence fits to the permittivity of the wall material (Brewster angle phenomenon). Vertical waves do not suffer a similar effect and will be therefore reflected (according to Kyritsi and Cox, 2001).

2. The average path loss exponent is below two $(\gamma<2)$. This means that the path loss inside of the measured building increases slower than in free space. The reason for that is that the rooms and hallways of the buildings serve as a kind of wave guides due to reflections at floors and ceilings (see also the previously mentioned subitem).

3. For a NLOS scenario the standard deviation is smallest if a horizontal wave is transmitted. A possible explanation for this phenomenon is the following: horizontal waves suffer less from reflections on walls (see the first subitem), so multipath fading becomes less important.

The results of Table 2 are independent from the used receiver antenna. Table 3 shows the standard deviation in dependency of the used receiver antenna (vertically polarized dipole or circular polarized patch antenna). Two points are remarkable:

1. The standard deviation is the smallest for the transmitter/receiver antenna combination linear to circular (or vice versa). This outcome confirms the theoretical considerations introduced in Sect. 1. 

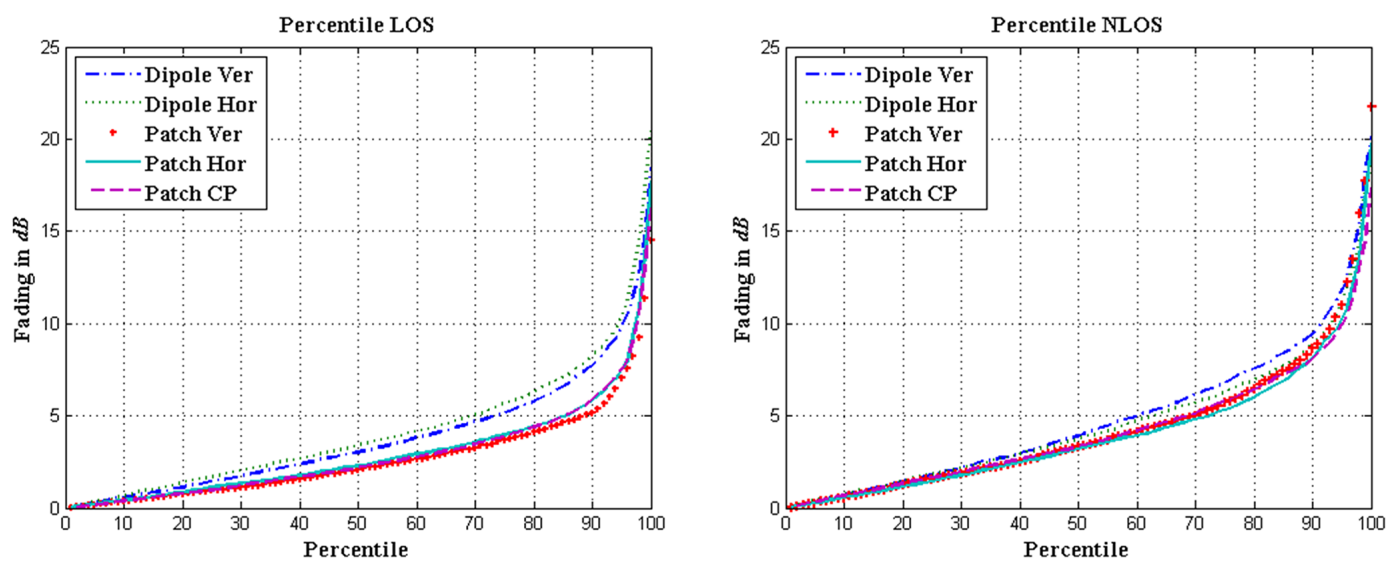

Figure 4. Percentile measure of fading (Dipole/Patch $=\mathrm{Rx}$ antenna; Ver/Hor/CP $=\mathrm{Tx}$ antenna).
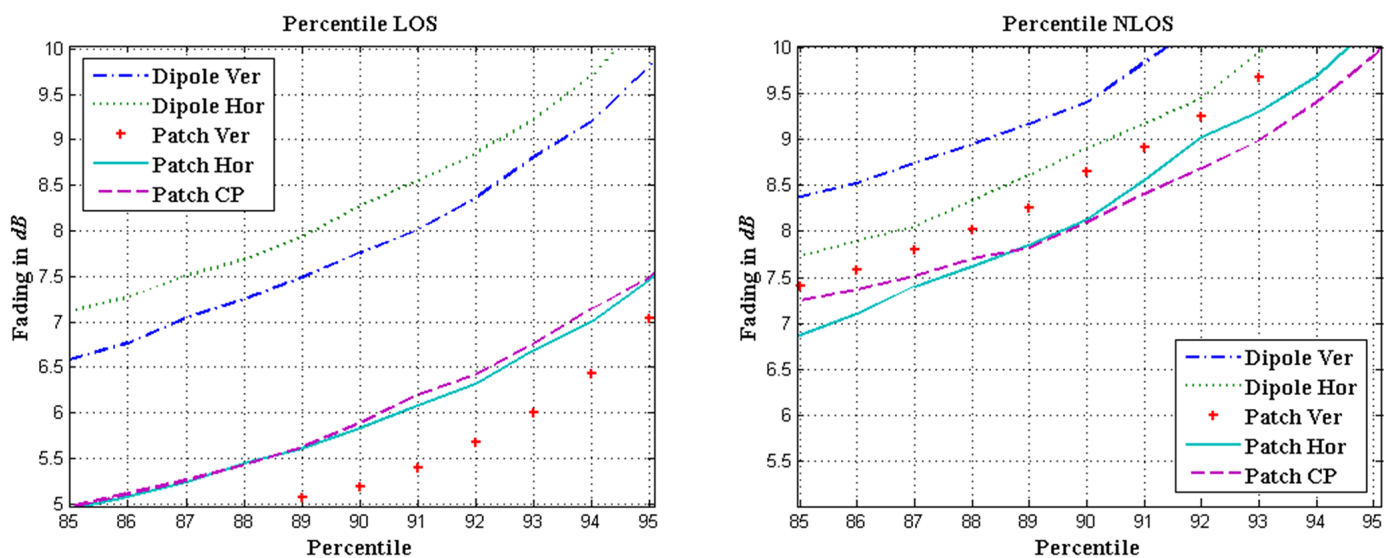

Figure 5. Percentile measure of fading, segment of the 90th percentile.

2. The combination circular/circular shows also good results, especially for LOS conditions. The explanation for this is that the energy of single-bounced reflected paths of the circular polarized transmitted wave disappears at the receiver since an odd number of reflections cause a reverse of the handedness of the circular polarized wave (Kajiwara, 1995).

A graphical representation of fading can be seen in Fig. 4. It shows a percentile measure of the encountered fading. Most measurement values of the patch antenna are below the according measurement values of the dipole. For example the 90th percentile (see Fig. 5) shows that $90 \%$ of the measurement values of the patch (LOS) are below a value of 5-6 dB, while $90 \%$ of the measurement values of the dipole are below a fading value of 7.5-8.5 dB. The same situation shows for a NLOS connection, the fading of $90 \%$ of the measurement values of the patch are below $8-9 \mathrm{~dB}$, while $90 \%$ of the values of the dipole are below 9-9.5 dB.

Two other papers which describe similar topics like this paper are e.g. Seidel and Rappaport (1992) and Kajiwara (1995). Kajiwara (1995) discusses different anti-multipath fading schemes using circular polarization under LOS conditions at $10 \mathrm{GHz}$. The results show that the mean amplitude fade level is reduced by $7-11 \mathrm{~dB}$ by using $\mathrm{CP}$ transmission/reception. The results by using CP to linear transmission/reception are slightly less good.

Seidel and Rappaport (1992) introduce a $914 \mathrm{MHz}$ path loss prediction model with a similar approach like in this paper. Different propagation scenarios were considered (e.g. grocery store, retail store and two office buildings). The path loss exponent and standard deviation was determined within a value range of $\gamma=1.81-5.04$ and $\sigma=4.3-$ $16.3 \mathrm{~dB}$, these relatively high values occur due to fact that the knowledge of the number of floors and walls between sender and receiver were not taken into account (see also Sect. 3). In relatively open environments (retail/grocery store) the path loss exponent is much smaller $\gamma=1.81-2.18$ which is similar to the values which were encountered in this paper. The standard deviation of the most scenarios $\sigma=4.3-8.7 \mathrm{~dB}$ is also similar to the values presented in this paper.

The paper Molisch et al. (2004) presents results for UWB (ultra wide band) channel models at $100-1000 \mathrm{MHz}$ and 
2-10 GHz. Even though UWB channel models are not fully comparable to the narrowband model which was used in this paper, the results show some similarities to the results presented in this paper. The path loss exponent and standard deviation was determined within a value range of $\gamma=1.63-1.79$ and $\sigma=0.83-2.22 \mathrm{~dB}$ for LOS conditions and $\sigma=2-3.51 \mathrm{~dB}$ for NLOS conditions (in the $2-10 \mathrm{GHz}$ band) for residential and office environments. The results for the $100-1000 \mathrm{MHz}$ band are only given for an officelike environment under NLOS conditions with $\gamma=2.4$ and $\sigma=5.9 \mathrm{~dB}$.

\section{Conclusion}

The results of a measurement campaign were presented. This measurement campaign was performed to create a measurement based fading model and to evaluate the possibility to fight fading using a circular polarized and a linear polarized antenna in an one-to-one transmission link within an indoor scenario.

The path loss exponent was measured within a range of values $\gamma=1.83-2$ and the standard deviation with mean value of $\sigma=4.24 \mathrm{~dB}$ for LOS conditions and $\sigma=5.67 \mathrm{~dB}$ for NLOS conditions. These values (especially the path loss exponents which is less or equal 2) seem to be in accordance with other studies presented in the previous chapter.

The method to combat fading using an optimized circular polarized truncated corner patch antenna has shown significant results. The results have shown that it is possible to mitigate fading by a notable amount, up to $3.5 \mathrm{~dB}$ for LOS and $1.5 \mathrm{~dB}$ for NLOS conditions (based on the 90th percentile), by replacing a linear polarized antenna (which is normally used in indoor SRD communication systems) with a circular polarized antenna. This is of practical importance since this is a very cost-efficient, space-saving and easy-to-realize method.

Acknowledgements. This project (HA-Project-Nr.: 344/12-34) is funded by LOEWE, Hesse's program of excellence in research.

Edited by: J. Götze

Reviewed by: three anonymous referees

\section{References}

Cox, D. C., Murray, R. R., Arnold, H. W., Norris, A., and Wazowicz, M.: Cross-polarization coupling measured for $800 \mathrm{MHz}$ radio trans-mission in and around houses and large buildings. Antennas and Propagation, IEEE T. Antenn. Propag., 34, doi:10.1109/TAP.1986.1143714, 1986.

Hashemi, H. and Tholl, D.: Analysis of the RMS delay spread of indoor radio propagation channels, Discovering a New World of Communications, IEEE International Conference, 2, 875-881, doi:10.1109/ICC.1992.268160, 1992.

Hernandez, M., Li, H.-B., Dotlic, I., Miura, R. (NICT), IEEE P802.15 Working Group for Wireless Personal Area Networks (WPANs): Channel models for TG8, IEEE P802.15-12-0459-000008, 2012.

Horvat, G., Rimac-Drlje, S., and Žagar, D.: Fade Depth Prediction Using Human Presence for Real Life WSN Deployment, Radioengineering, 22, 758-769, 2013.

Kajiwara, A.: Line-of-Sight Indoor Radio Communication Using Circular Polarized Waves, IEEE T. Veh. Technol., 44, 487-493, 1995.

Kyritsi, P. and Cox, C.: Propagation characteristics of horizontally and vertically polarizedelectric fields in an indoor environment: simple model and results, Vehicular Technology Conference, IEEE VTS 54th, 3, 1422-1426, doi:10.1109/VTC.2001.956431, 2001.

Molisch, A.: Wireless Communications, John Wiley \& Sons Ltd., second edition, p. 27, 2011.

Molisch, A., Balakrishnan, K., Chong, C.-C., Emami, S., Fort, A., Karedal, J., Kunisch, J., Schantz, H., Schuster, U., and Siwiak, K.: IEEE P802.15.4, 662-703, IEEE 802.15.4a channel model - final report, http://ieee802.org/15/pub/04/ 15-04-0662-01-004a-channel-model-final-report-r1.pdf, last access: 2 September 2014, 2004.

Schwarzbeck Mess-Elektronik: XSLP 9142 Kreuzpolarisierte Breitband UHF-SHF Log.-Per. Messantenne Dual Polarized UHFSHF Broadband Log.-Per. Test-Antenna $800 \mathrm{MHz}$... 3 (5) GHz, http://www.schwarzbeck.com/Datenblatt/manx9142.pdf, last access: 22 July 2014, 2013.

Seidel, S. and Rappaport, T.: $914 \mathrm{MHz}$ path loss prediction for indoor wireless communication in multifloored buildings, IEEE T. Antenn. Propag., 40, 207-217, 1992.

Vireerackody, K.: Characteristics of a simulated fast fading indoor radio channel, Vehicular Technology Conference, 43rd IEEE, 231-235, doi:10.1109/VETEC.1993.507051, 1993. 